\title{
Primitive neuroectodermal tumor of the esophagus with metastasis in the pineal gland $\square$
}

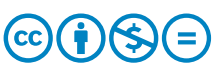

\author{
Authors \\ Leonardo Blas Jhon, Paloma Sánchez-Fayos, Maria Jesus Martín Relloso, Daniel Calero Barón, Juan Carlos Porres
} Cubero

Institution

Endoscopy Department, University Hospital Fundacion Jimenez Diaz, Madrid, Spain

submitted 10.10 .2018

accepted after revision 21.6.2019

Bibliography

DOI https://doi.org/10.1055/a-0977-2614 |

Endoscopy International Open 2019; 07: E1163-E1165

(c) Georg Thieme Verlag KG Stuttgart · New York

eISSN 2196-9736

Corresponding author

Leonardo Blas Jhon, Hospital Universitario Fundacion

jimenez Diaz, Aparato Digestivo, Avenida Reyes Católicos,
Madrid 28040, Spain

Fax: +34-91-5448014

solyaire_3@hotmail.com

\section{ABSTRACT}

Primitive neuroectodermal tumors (PNET) are very rare tumors that belong to a family of malignant neoplasms of tiny round cells which are derived from the neural crest. This report discusses a rare case of an adult woman with esophageal PNET, confirmed by immunohistochemistry, that presented with metastasis to the pineal gland. To our knowledge, this is the first case report of a PNET with these features. Despite surgery and chemotherapeutic treatment, our case has shown disease progression.

\section{Introduction}

Primitive neuroectodermal tumors (PNET) are very rare tumors that belong to a family of malignant neoplasms of tiny round cells which are derived from the neural crest. There are three types: PNET of the central nervous system, PNET of the autonomic nervous system, and peripheral PNET. The classification and terminology of this third group are complicated and controversial. The most frequent occurrences in the peripheral PNET group are the peripheral neuroepitheliomas and Ewing sarcomas (osseous and extraosseous), which are considered to be the same neoplasm but with a different grade of differentiation. This report discusses a rare case of an adult woman with esophageal PNET that presented with metastasis to the pineal gland.

\section{Clinical case}

A 49-year-old woman presented with a headache and diplopia. There was nothing unusual in her medical history. Neurological examination revealed vertical gaze palsy, convergence nystagmus, and decreased light reflex (Parinaud's syndrome).

A head CAT scan was performed and indicated a tumor of the third ventricle with incipient hydrocephalus. Cerebral NMR was carried out to improve characterization ( $\triangleright$ Fig. 1) and defined a solid 17-mm mass in the pineal region. After administration of IV contrast, peripheral calcification and vivid enhancement could be seen indicating active triventricular obstructive hydrocephalus with signs of transependymal resorption. There were no signs of leptomeningeal uptake at that time.

With the finding of a pineal tumor, a craniectomy of the posterior fossa was performed with a supracerebellar infratentorial approach to the pineal region and an external ventricle drain was placed. The pineal region and third ventricle were accessed by removing the tumor mass using microsurgery and the StealthStation S7 Surgical Navigation System (Medtronic, Louisville, CO, USA) with curative intent.

Histopathological examination of the tumor revealed proliferations with high cellular density forming lobes. The cells showed scant cytoplasm, elongated atypical nuclei and with abundant mitotic figures with up to 10 mitoses in 1 high-power field. Foci of necrosis and areas with abundant electrocautery artifacts were recognized. These areas had a lower cellular density, and peripheral calcification suggestive of a pineoblastoma. Immunohistochemistry showed a patchy expression of synaptophysin, a diffuse and intense expression of CD99, and occasional expression of cytokeratins AE1/AE3 and CAM 5.2. Fluorescence in situ hybridization analysis (FISH) revealed reciprocal 


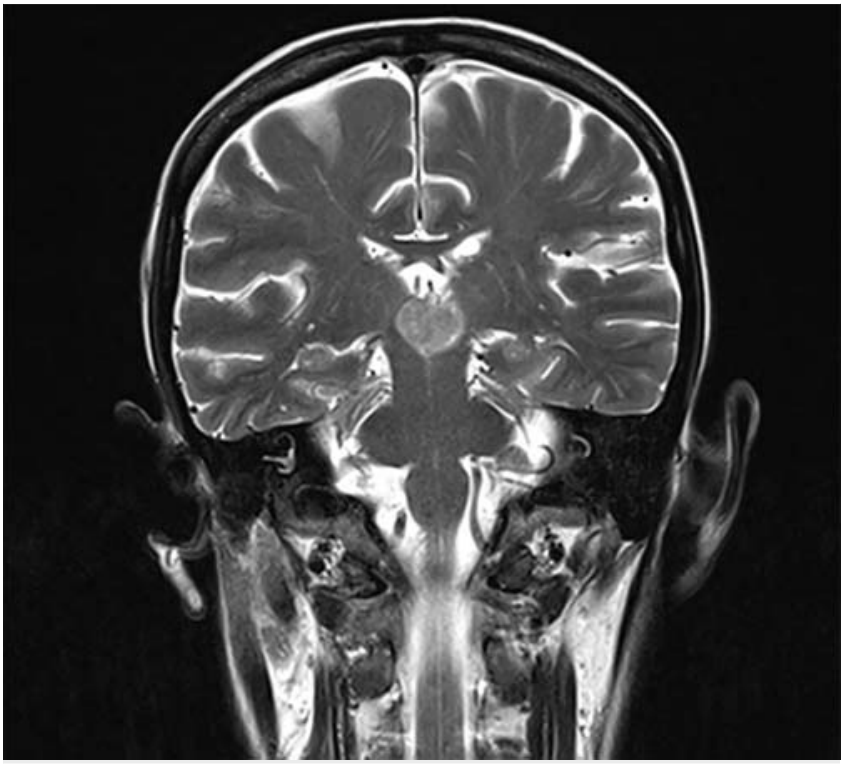

- Fig. 1 Coronal post-contrast T1-weighted MR image showing an avidly enhancing mass in the pineal region, with peripheral calcification and resultant hydrocephalus.

translocation of EWSR1 $\mathrm{t}(22 \mathrm{q} 12)$. The pathological report concluded that the lesion was PNET located in the pineal gland with atypical nuclei, high mitotic activity and necrosis, and with a Ki67 index of $80 \%$, compatible with pineoblastoma. However, it was not possible to discount a metastatic origin for the peripheral PNET.

The patient's condition in the postoperative period was satisfactory. One week later, a cranial CT scan was performed showing post-surgical changes. The ventricular size remained stable. The periventricular hypodensity persisted although to a greater extent now than on preoperative images and suggestive of residual transependymal exudation. No other alterations were noted.

Subsequently, with the results of the pathological report, the case was presented to the neuro-oncological tumor committee and it was decided to request a thoraco-abdomino-pelvic CT scan ( $\triangleright$ Fig. 2 ) as a follow-up study. This revealed a circumferential thickening of the walls of the middle third of the esophagus (ca $3.7 \mathrm{~cm}$ in length). No transmural involvement was observed. The CT findings probably indicated the presence of an esophageal neoplasm; however, the patient did not present any digestive symptoms at that time.

To clarify the situation, we performed an upper endoscopy ( $>$ Fig. 3, - Video 1). A longitudinal and deep esophageal ulcer with dimensions of about $5 \times 1 \mathrm{~cm}$ was present at a depth of 25 to $30 \mathrm{~cm}$ with background fibrin that presented mamelonated edges of hard consistency during biopsy. The report on the pathologic anatomy ( $\triangleright$ Fig.4a) showed an epithelium infiltrated by a poorly differentiated neoplasm characterized by round, very small cell nests with hyperchromatic nuclei, but with no cytoplasm and with moderate desmoplasia. The immunohistochemical study ( $\triangleright \mathbf{F i g} \mathbf{4} \mathbf{4 b}$ ) reported a diffuse expression of vimentin and CD99 and occasional expression of cytokeratin

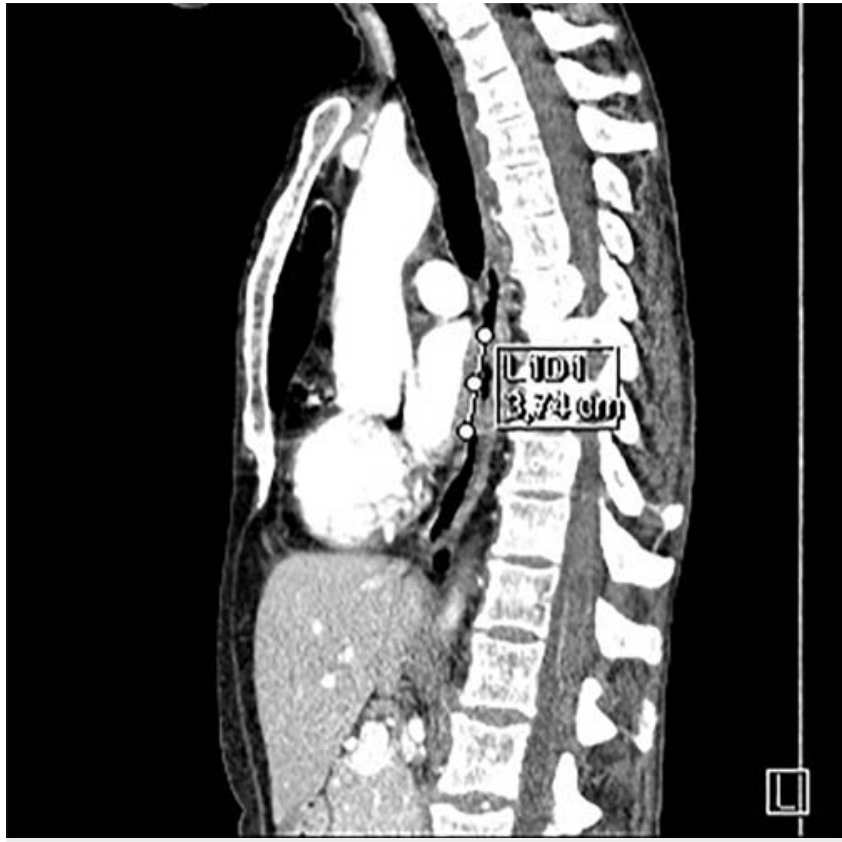

Fig. 2 Sagittal CT image showing thickening of the wall of the middle esophagus.

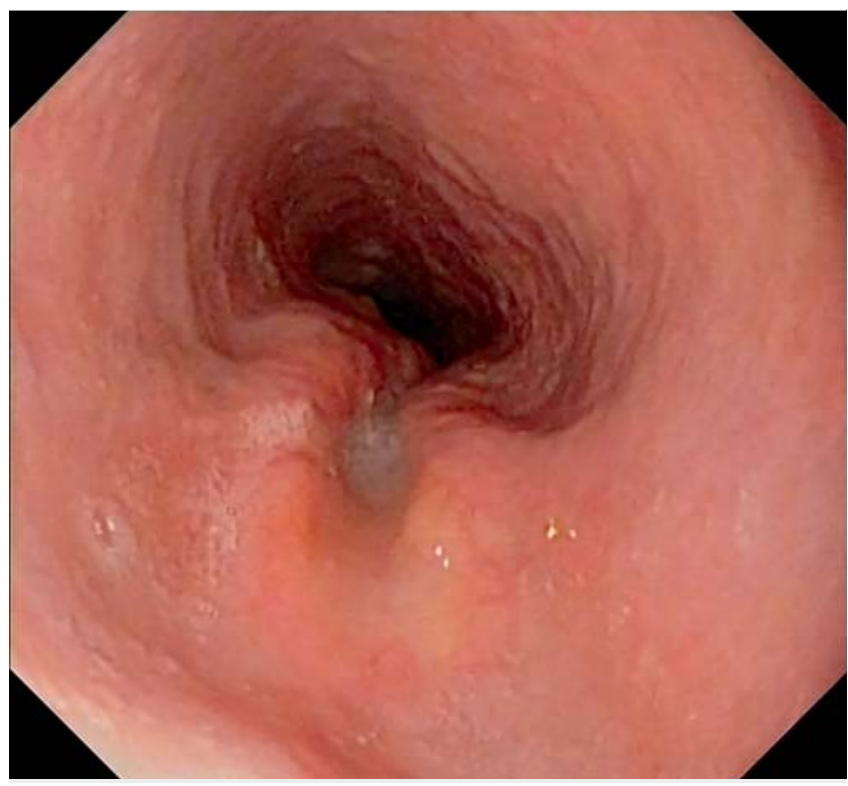

- Fig. 3 Upper endoscopy showing an esophageal ulcer with raised and indurated edges that may indicate primary esophageal cancer.

AE3. The MIB-1 proliferation index was $100 \%$. The cytogenetic analysis was not useful as it did not detect signals corresponding to the genes studied due to poor cellular DNA preservation. These findings indicated esophageal PNET, with an immunohistochemical profile very similar to that of the resected tumor found in the pineal region and discussed earlier.

MRI of the complete neuraxis was also performed as a control and which indicated laminar dura mater enhancement (possibly postoperative), changes related to partial resection 


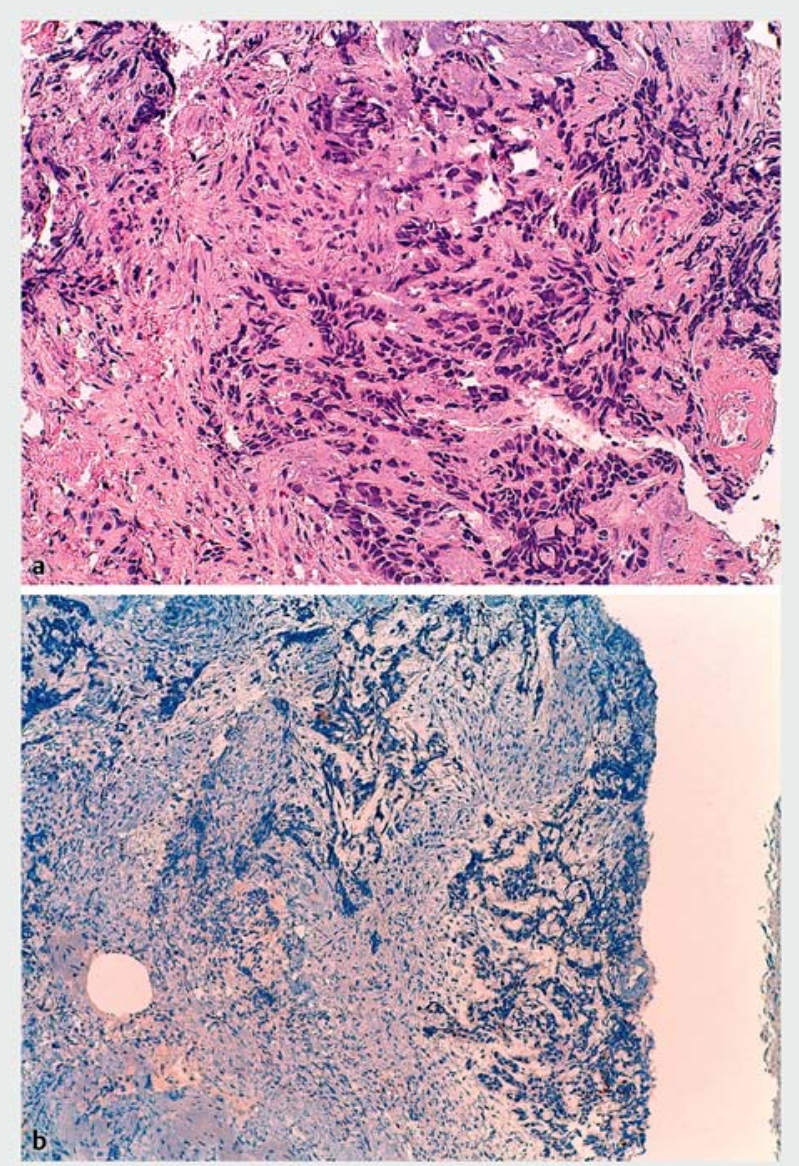

- Fig. 4 Histologic appearance of the tumor. a Tumor cells with uniform, mildly atypical nuclei, granular chromatin, and scarce cytoplasm. Pseudorosette-like structures are also seen (hematoxylin and eosin staining). b Immunohistochemistry of tumor cells showing immunoreactivity for CD99 on the membrane of all tumor cells.

of the mass in the pineal region, with a large remnant mass, but without signs of bleeding. Disseminated pathologic areas with IV uptake of contrast medium were also seen indicating leptomeningeal carcinomatosis suggesting probable tumoral dissemination, with the absence of space-occupying lesions.

The case was discussed by the neuro-oncological tumor committee where it was decided that vincristine, adriamycin, cyclophosphamide (VAC) alternating with ifosfamide and etoposide (IE) chemotherapy must be initiated. The patient is currently receiving chemotherapy with good tolerance; however, at the last control, there was evidence of tumor progression

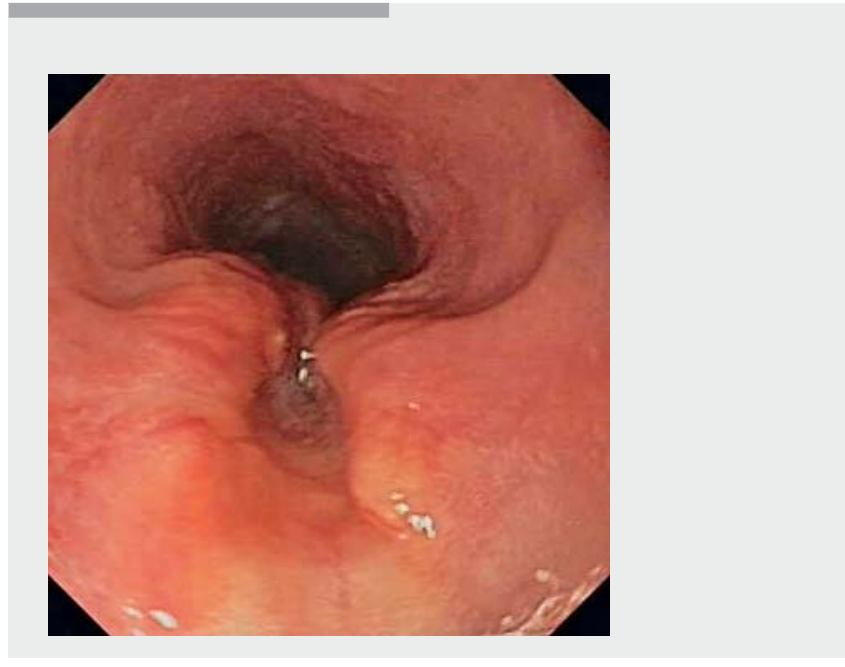

$\triangle$ Video 1 Upper endoscopy showing a longitudinal and deep esophageal ulcer with dimensions of about $5 \times 1 \mathrm{~cm}$ and present at a depth of 25 to $30 \mathrm{~cm}$.

(regrowth of tumoral remnants in the pineal gland and worsening of the leptomeningeal carcinomatosis), so, depending on the results of chemotherapy, the next stage may be radiotherapy treatment.

In conclusion, we report the unusual case of a 49-year-old woman with esophageal PNET, confirmed by immunohistochemistry, that presented with metastasis to the pineal gland. To our knowledge, this is the first case report of a PNET with these features. PNET are very rare neoplasms and are highly aggressive. In all cases, they appear as tiny round cells, slightly differentiated and with a chromosomal translocation characteristic of the EWS gene. The treatment protocol for this type of tumor includes surgery, multi-agent chemotherapy and, in some cases, radiotherapy. After treatment, patients must be followed for years due to the possibility of recurrence or metastasis which is very common for this type of tumor. Despite surgery and chemotherapeutic treatment, our case has shown disease progression. In general, PNET are considered to have an unfavorable prognosis. In addition, the rarity of these tumors, and the lack of published cases make it hard to establish a more precise treatment protocol.

\section{Competing interests}

None 